\title{
Antibacterial Effect of Ginseng/polyaniline Encapsulated in Poly(Lactic-co-glycolic Acid) Microcapsules Coating on Stainless Steel 316L
}

\author{
Siti Khadijah Lukman ${ }^{1}$ and Syafiqah Saidin ${ }^{1,2, a}$ \\ ${ }^{1}$ School of Biomedical Engineering \& Health Sciences, Faculty of Engineering, Universiti Teknologi Malaysia, 81310 \\ UTM Johor Bahru, Johor, Malaysia. \\ ${ }^{2}$ IJN-UTM Cardiovascular Engineering Centre, Institute of Human Centered Engineering, Universiti Teknologi \\ Malaysia, 81310, UTM Johor Bahru, Johor, Malaysia.
}

\begin{abstract}
Implant infection is one the current complications of implant restoration. Incorporation of a material that possesses antibacterial property is crucial in combating implant infection. In this study, polyaniline (PANI) is incorporated in ginseng encapsulated poly (lactic-co-glycolic acid) (PLGA) microcapsules to equip the microcapsules with an antibacterial effect. The microcapsules are intended for drug delivery purpose as the ginseng is comprised of abundant therapeutic values and the PLGA is known for its degradation property. The microcapsules were coated on stainless steel 316L using an electro-deposition technique. The chemical composition, morphology and antibacterial activity of the samples were analyzed through ATR-FTIR, SEM and bacterial count test. A greater volume of microcapsules was coated on the stainless steel 316L with the addition of PANI through the appearance of intense ginseng $(\mathrm{O}-\mathrm{H}$ and $\mathrm{C}=\mathrm{C}$ bands) and PLGA $(\mathrm{C}=\mathrm{O})$ peaks on the ATR-FTIR spectra and through the visualization of spherical morphology of microcapsules on the pre-treated metal. The incorporation of PANI also has induced the antibacterial efficacy of the microcapsules to 87.64\%. Therefore, PANI has served as an antibacterial agent that could be useful in the development of implant coating materials while driving the formation of ginseng encapsulated PLGA microcapsules.
\end{abstract}

\section{Introduction}

Implant is a temporary or permanent substitute to replace dysfunction organs. Despite of continuous advancement in metallic implant techniques and materials, implant infection is among the current complications of implant restoration in various medical fields such as dentistry [1], cardiovascular [2] and orthopedics [3]. The treatment of implant infection is time-consuming and costly which might lead to the replacement of failure infected implants [3]. Hence, providing an antibacterial property on implant materials is crucial as one of the approaches in addressing this complication.

Polyaniline (PANI) is a conductive polymer that also possesses an antibacterial effect [4]. It receives great attention due to its attractive electrical properties through its modification in oxidation and protonation states $[5,6]$. Polyaniline exists in different forms such as fully oxidized pernigraniline

\footnotetext{
${ }^{a}$ Corresponding author: syafiqahsaidin@ biomedical.utm.my
} 
base, half-oxidized emeraldine base and fully reduced leucoemeraldine [7]. The antibacterial properties of PANIs [4], PANI doped with natural acid [8] and PANI/CZO nanocomposites [9] have been explored. The antibacterial activities of different forms of PANI against four gram positive, five gram negative bacteria and fungus Candida albicans were also evaluated by Dhivya et al. has shown good antibacterial efficacies [10]. The mechanism of antibacterial effects of PANI also been discussed [10].

Poly (lactic-co-glycolic acid) (PLGA) is another polymer which is widely used in implant coating material that effective in controlling material/drug degradation [11]. It has been approved by Food and Drug Administration (FDA) to be restored in human [12]. In this study, PLGA was used to encapsulate ginseng where ginseng has abundant therapeutic benefit such as diabetic inhibitory [13], antioxidant activities [14] and anti-inflammatory effects [15]. Incorporation of PANI in the ginseng encapsulated microcapsules will further enhancing the therapeutic benefit of the microcapsules by providing a reservoir for antibacterial release.

Therefore, PANI was incorporated into ginseng encapsulated PLGA microcapsules to be coated on stainless steel 316L (SS316L) using an electro-deposition technique to equip the coating with antibacterial property. The chemical composition and morphology of the samples were characterized principally by using an attenuated total reflectance-Fourier transform infrared spectroscopy (ATRFTIR) and scanning electron microscopy (SEM), respectively. The antibacterial efficacy of the samples was also evaluated through a bacterial count test.

\section{Materials and Methods}

\subsection{Materials}

Poly(lactic-co-glycolic acid) with 85:15 lactide/glycolide ratio and inherent viscosity of $0.63 \mathrm{dL} / \mathrm{g}$ was supplied by LACTEL Absorbable Polymers, UK. While ginseng extract was purchased from Dalian Hongjiu Biotech, China. Polyaniline (emeraldine base) with a molecular weight of 20,000 and sodium chloride $(\mathrm{NaCl})$ were acquired from Sigma Aldrich, USA. Dichloromethane (DCM), fully hydrolyzed polyvinyl alcohol (PVA) with a molecular weight of 30,000 and 30\% hydrogen peroxide $\left(\mathrm{H}_{2} \mathrm{O}_{2}\right)$ were obtained from Merck KGaA, Germany. Acetone, hydrofluoric acid (HF), methanol, glycerol, phosphoric acid $\left(\mathrm{H}_{3} \mathrm{PO}_{4}\right)$ and nitric acid $\left(\mathrm{HNO}_{3}\right)$ were bought from Friendemann Schmidt, Western Australia. A thin foil medical grade stainless steel 316L with $0.25 \mathrm{~mm}$ thickness was obtained from Goodfellow Cambridge Limited, Huntingdon, England.

\subsection{Microcapsules Fabrication}

Ginseng/PANI was encapsulated in PLGA microcapsules through a double emulsion solvent evaporation method [16]. In the first stage, $400 \mathrm{mg}$ PLGA and $1 \mathrm{mg}$ PANI were dissolved in $10 \mathrm{~mL}$ of DCM and $30 \mathrm{mg}$ ginseng extract was dissolved in $1 \mathrm{~mL}$ of distilled water, separately. In achieving water-in-oil (W/O) emulsion, both suspensions were emulsified with a homogenizer for 6 min at $20,000 \mathrm{rpm}$ in an ice bath. In order to achieve water-in-oil-in-water $(\mathrm{W} / \mathrm{O} / \mathrm{W})$ emulsion, the resulting $\mathrm{W} / \mathrm{O}$ emulsified mixture was instilled into $60 \mathrm{~mL}$ of $1.25 \% \mathrm{w} / \mathrm{v}$ PVA solution and homogenized for $10 \mathrm{~min}$ at 20,000 rpm in an ice bath.

The mixture was stirred overnight at room temperature to evaporate the DCM. The mixture was then centrifuged at $10,000 \mathrm{rpm}$ for $30 \mathrm{~min}$ at $4^{\circ} \mathrm{C}$. The centrifugation cause noticeable appearance of microcapsules. The microcapsules were washed once with $0.9 \% \mathrm{NaCl}$ and twice with distilled water. Finally, the ginseng/PANI encapsulated PLGA microcapsules were stored at $5^{\circ} \mathrm{C}$ prior to use. The processes were repeated without the addition of PANI to fabricate ginseng encapsulated PLGA microcapsules.

\subsection{Sample Preparation}




\subsubsection{Pre-treatment}

Medical grade stainless steel 316L foil was cut into square shape of $10 \mathrm{~mm} \times 10 \mathrm{~mm}$ and undergoes several pre-treatment processes [17] included ultrasonic cleaning, electro-polishing and acid-dipping. The metals were each ultrasonic cleaned in acetone, DI water and methanol for $10 \mathrm{~min}$. Then, the cleaned metals were electro-polished for $3 \mathrm{~min}$ at $1.5 \mathrm{~A}$ in an electrolyte solution $(50 \%$ glycerol, $15 \%$ DI water and $35 \% \mathrm{H}_{3} \mathrm{PO}_{4}$ ). Acid-dipping was done by dipping the electro-polished metals in an acid solution $\left(10 \% \mathrm{HNO}_{3}, 2 \% \mathrm{HF}\right.$ and $88 \%$ DI water) for $30 \mathrm{sec}$ to remove the remaining electro-polishing ions that adhered on the metal surface. The pre-treated metals were then rinsed with DI water, followed by drying using an air compressor.

\subsubsection{Microcapsules Deposition}

The ginseng/PANI encapsulated PLGA microcapsules and the ginseng encapsulated PLGA microcapsules, denoted by PLGA/g/PN and PLGA/g, respectively, were deposited on the pre-treated metals using an electrodeposition technique following the procedures by Wang et al. [18] with some alterations. An electrolyte solution was prepared by adding $1 \% \mathrm{w} / \mathrm{v}$ microcapsules into DI water. The conductivity of the electrolyte was improved with the addition of $0.15 \mathrm{M} \mathrm{NaCl}$ and $100 \mathrm{mM} \mathrm{H}_{2} \mathrm{O}_{2}$. The electrolyte was stirred for $30 \mathrm{~min}$ at room temperature. The microcapsules were electro-deposited using a direct current (DC) power supply (72-8690A, TENMA, Japan) in a laminar flow. The pretreated metal was clasped at the cathode while a reference electrode was clasped at the anode. Both cathode and anode were then immersed in an electrolyte solution at room temperature. The electrodeposition was conducted at $2 \mathrm{~mA}$ current density for $40 \mathrm{sec}$. Finally, the coated metals were washed with DI water and dried in a vacuum desiccator prior to analyses.

\subsection{Characterization analyses}

\subsubsection{Chemical Composition Analysis}

The PLGA/g/PN, PLGA/g and pre-treated metal were characterized using attenuated total reflectanceFourier transform infrared spectroscopy (ATR-FTIR). The surface composition of the samples was determined by ATR-FTIR (Nicoler iD5, Thermo Scientific, USA) using ZnSe crystal at $4 \mathrm{~cm}^{-1}$ resolution. The spectra were recorded within $500-4000 \mathrm{~cm}^{-1}$ frequency range and collected at 32 average scans.

\subsubsection{Morphological Analysis}

The microstructure and morphology of PLGA/g/PN, PLGA/g and pre-treated metal were visualized using a tabletop SEM (Hitachi, TM300, Japan), viewing at an accelerating voltage of $15 \mathrm{kV}$, under magnification of $1000 \times$ and $3000 \times$. A thin layer of gold was sputtered on the microcapsule coating to avoid charging under electron beam, prior to the SEM analysis.

\subsection{Antibacterial analysis}

The antibacterial activity of the samples was evaluated with Gram-negative Eschericia. coli (E. coli, ATCC 25922) through a quantitative analysis. The pre-treated metal, PLGA/g and PLGA/g/PN were sterilized with ultraviolet (UV) light on each surface for $30 \mathrm{~min}$. The samples were then immersed in 5 $\mathrm{mL}$ of bacterial suspension that contained $1 \times 10^{6}$ cells $/ \mathrm{mL}$ bacteria. All inoculated samples were incubated for $3 \mathrm{~h}$ in a shaking incubator at $200 \mathrm{rpm}$. After incubation, the samples were removed and rinsed two times with phosphate buffer saline (PBS) to remove non-attached bacteria. The samples were placed in a new sterile centrifuge that contained $5 \mathrm{~mL}$ of Luria-Bertani (LB) broth. The adhered 
bacteria on the sample surfaces were detached using a vortex for $30 \mathrm{sec}$. The bacterial suspensions were diluted to six dilution series. A drop plate method was then used to grow the bacteria on LB agar. The method was performed by pipetting $10 \mu \mathrm{L}$ of each dilution on LB agar to be incubated for $24 \mathrm{~h}$ at $37^{\circ} \mathrm{C}$. The colony-forming units (CFUs) were counted manually to obtain the number of adherent bacteria. Equation 1 was finally used to calculate the bactericidal ratio.

$$
\text { Bactericidal ratio }=\frac{\left(C F U_{\text {control }}-C F U_{\text {experiment }}\right)}{C F U_{\text {control }}} \times 100
$$

\section{Results and Discussion}

\subsection{Characterization analyses}

\subsubsection{Chemical Composition Analysis}

Figure 1 shows the ATR-FTIR spectra of pre-treated metal, PLGA/g and PLGA/g/PN. No identical peak was depicted on the pre-treated metal, defining the pre-treated metal surface was clear from apparent functional group. The presence of PLGA and ginseng were validated on the PLGA/g/PN and PLGA/g when C-O peaks were noticed at 1089 and $1182 \mathrm{~cm}^{-1}$ due to the existence of $\mathrm{C}-\mathrm{O}$ functional groups. However, another identical peak of PLGA and ginseng was only noticed on the PLGA/g/PN, denoted by $\mathrm{C}-\mathrm{H}$ peak at $1459 \mathrm{~cm}^{-1}$.

Strong peak of carbonyl $\mathrm{C}=\mathrm{O}$ stretching vibration at $1741 \mathrm{~cm}^{-1}$ was also recorded only on the PLGA/g/PN, demonstrating the composition of PLGA. Meanwhile, the composition of ginseng was clarified by the appearance of strong and broad peak of $\mathrm{O}-\mathrm{H}$ band $\left(3000-3500 \mathrm{~cm}^{-1}\right)$ on both PLGA/g/PN and PLGA/g. A medium peak of $\mathrm{C}=\mathrm{C}$ stretch alkene was also observed only on the $\mathrm{PLGA} / \mathrm{g} / \mathrm{PN}$ at $1644 \mathrm{~cm}^{-1}$, presenting the functional group of ginseng. However, the presence of PANI on the PLGA/g/PN was not identified clearly as some peaks such as $\mathrm{N}-\mathrm{H}, \mathrm{C}-\mathrm{N}$ and $\mathrm{C}=\mathrm{C}$ from the PANI emeraldine compound [10,19] might overlay with other peaks arising from the PLGA and ginseng. From the results of ATR-FTIR, the ginseng/PANI encapsulated PLGA microcapsules were intensely deposited on the pre-treated metal with the facilitation of PANI due to the appearance of strong PLGA and ginseng functional groups on the metal surface.

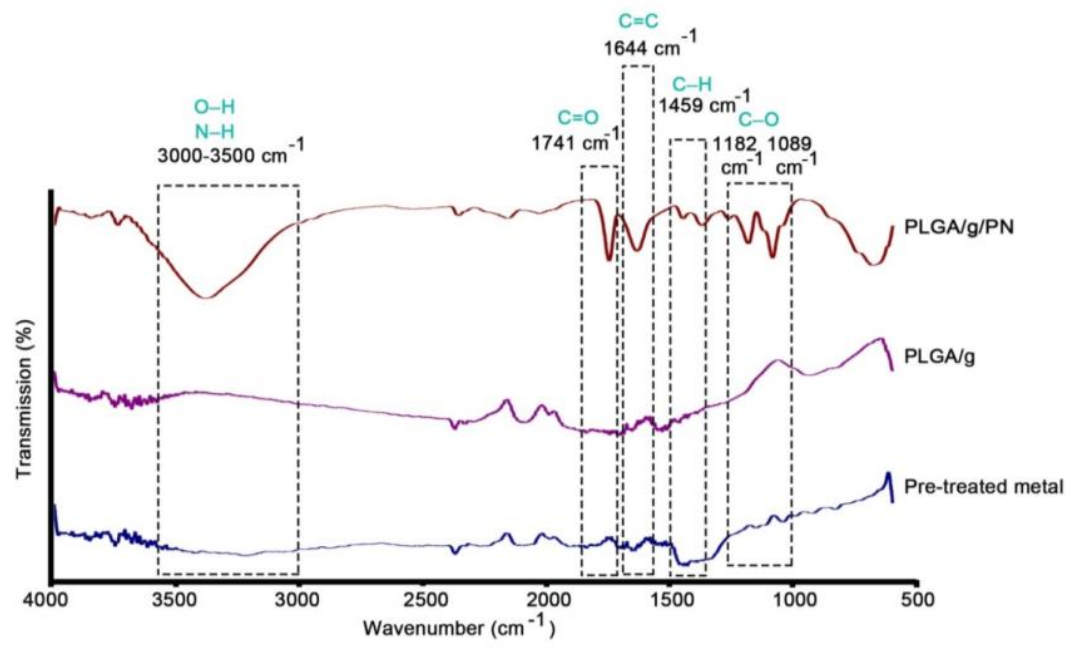

Figure 1. ATR-FTIR spectra of pre-treated metal, PLGA/g and PLGA/g/PN. 
Figure 2 shows the morphology of pre-treated metal, PLGA/g and PLGA/g/PN. A clear and smooth surface was observed on the pre-treated metal due to the pre-treatment processes of ultrasonic cleaning, electropolishing and etching. The passive layers of stainless steel 316L were dissolved during the electropolishing caused the removal of micro-scratch and flaws. While the etching was intended to remove the phosphate layer adhered on the metal surfaces [20]. There was a few formation of microcapsules on the PLGA/g where greater volume of microcapsules was visualized on the PLGA/g/PN. The incorporation of PANI played a role in the attachment of microcapsules on the pretreated metal where this material acted as a conductive agent [21] in driving the movement of microcapsules towards the metal surfaces during the electro-deposition process.
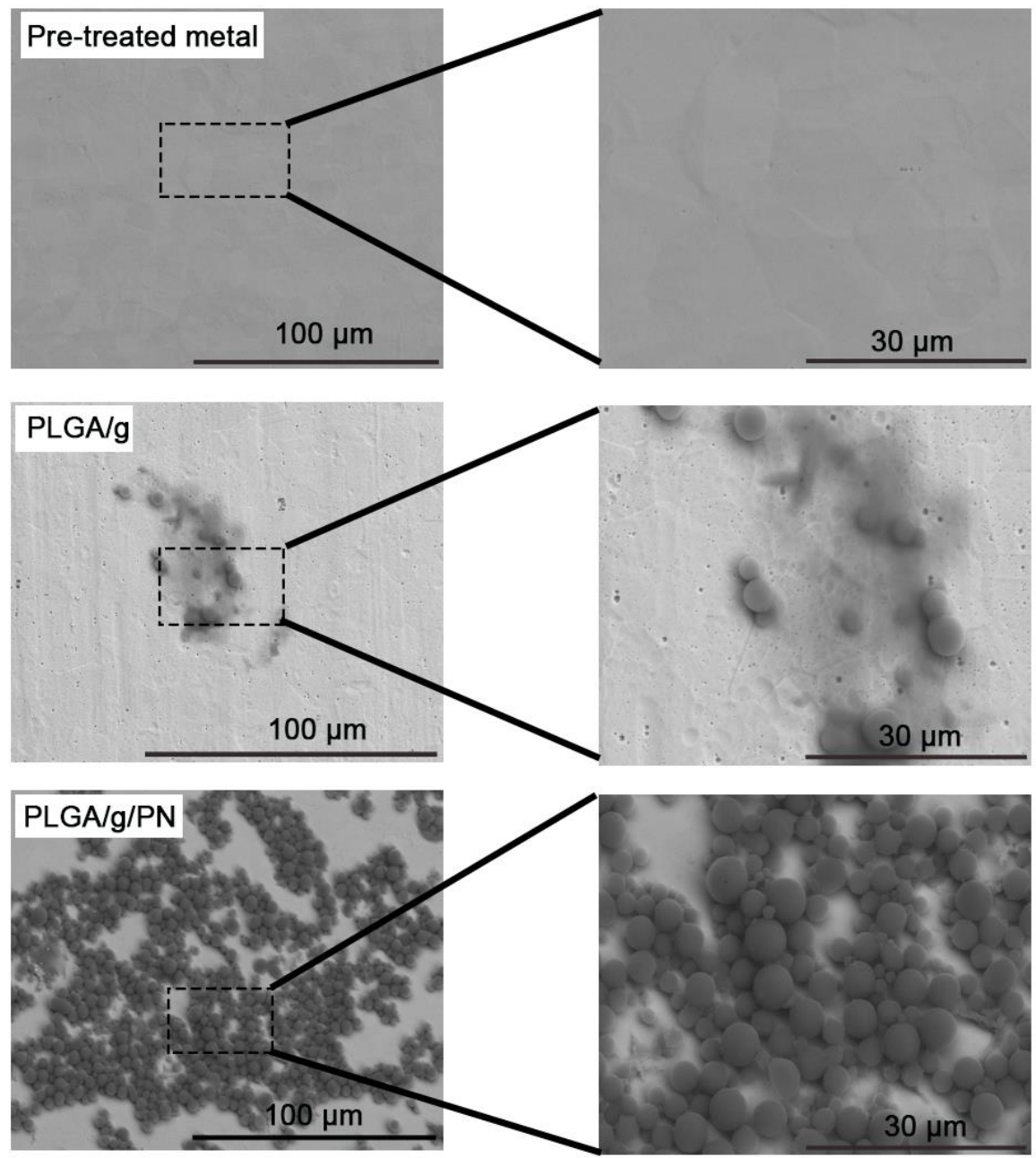

Figure 2. SEM images of pre-treated metal, PLGA/g and PLGA/g/PN.

\subsection{Antibacterial Analysis}

The data of antibacterial quantitative analysis were listed in Table 1 while the antibacterial activity of the samples on gram negative bacteria, E. coli was shown in Figure 3. These results were based on the bacteria adhered on the metal surfaces. Evidently, it was indicated that the pre-treated metal has no antibacterial effect. The bactericidal ratio of the PLGA/g/PN (87.64\%) against $E$. coli was higher than the PLGA/g (37.45\%). The presence of PANI in the PLGA/g/PN killed the adherent $E$. coli even 
though the surfaces might have more bacterial attachment due to the rough structure. A study by Nanlin et al. [4] stated that the bacterial death occurred due to acidic dopants on the molecular chain of PANI that reacted with the bacteria. Besides, the electrostatic of PANI molecules also played a role in providing the antibacterial effect [4].

Table 1. CFU/mL and bactericidal ratio of E. coli on pre-treated metal, PLGA/g and PLGA/g/PN

\begin{tabular}{|l|c|c|}
\hline Sample & CFU $\left(\mathbf{1} \times \mathbf{1 0} \mathbf{6}^{\mathbf{6}}\right.$ cells/mL) & Bactericidal ratio (\%) \\
\hline Pre-treated metal & $2.67 \pm 1.53$ & 0.00 \\
\hline PLGA/g & $1.67 \pm 0.57$ & 37.45 \\
\hline PLGA/g/PN & $0.33 \pm 0.57$ & 87.64 \\
\hline
\end{tabular}

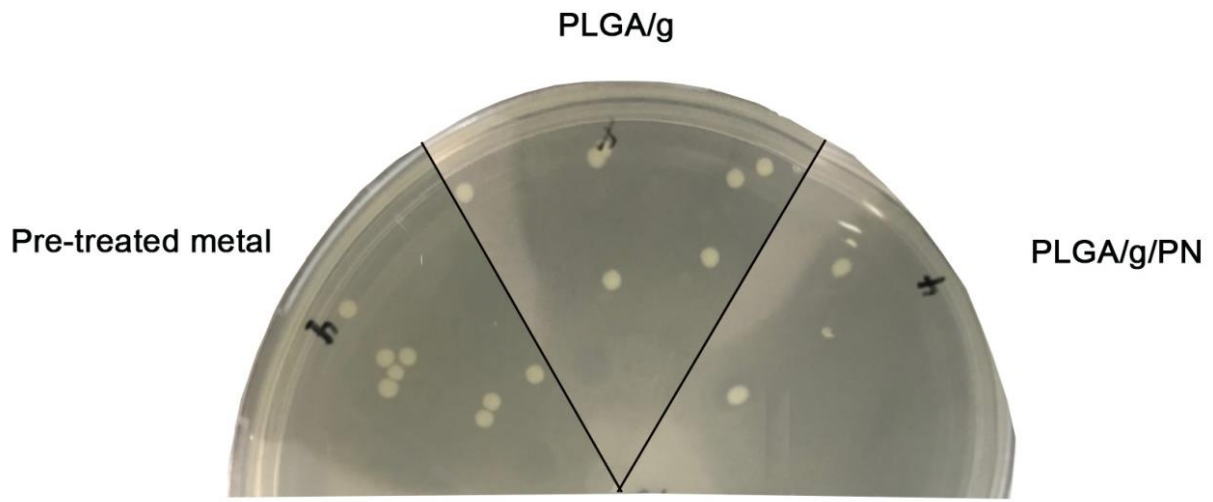

Figure 3.The antibacterial activity of the samples on Gram-negative Eschericia. Coli.

\section{Conclusion}

The incorporation of PANI in the ginseng encapsulated PLGA microcapsules has served the microcapsules with antibacterial effects. The microcapsules were coated onto stainless steel $316 \mathrm{~L}$ using electrodeposition technique. The formation of microcapsules was proved through ATR-FTIR and SEM images, where the microcapsules successfully deposited onto metal surface. It also provides antibacterial effects that may be usable in metallic implants when the bactericidal ratio on microcapsules incorporated PANI shows higher than without the addition of PANI. Therefore, metallic implant equipped with PANI will reduce the probability of infection and inhibit the bacterial growth while inducing the formation of ginseng encapsulated PLGA microcapsules.

\section{Conflict of Interest}

There is no conflict of interest.

\section{Acknowledgement}

This study was supported by Malaysian Ministry of Higher Education through a Research University Grant (RUG) Tier 1 [Q.J130000.2545.18H40]. The authors would like to appreciate Tan Sri Ainuddin Wahid scholarship and thank Mohamad Amin Jumat for his contribution in conducting antibacterial analysis. 


\section{References}

1. Y. Yu, G. Jin, Y. Xue, D. Wang, X. Liu, J. Sun, Acta. Biomater. 49, 590-603 (2017)

2. R. Martin, E. Paul, K. E. P. G. Richard, F. Reno, W. Maja, Pacing Clin. Electrophysiol. 38, 247$253(2015)$

3. C.W. Chan, L. Carson, G. C. Smith, A. Morelli, S. Lee, Appl. Surf. Sci. 404, 67-81 (2017)

4. N. Shi, X. Guo, H. Jing, J. Gong, C. Sun, K. Yang, J. Mater. Sci. Technol. 22, 289-290 (2006)

5. A. Mostafaei, A. Zolriasatein, Pro. Nat. Sci-Mater. 22, 273-280 (2012)

6. S.I. Abd Razak, I.F. Wahab, F. Fadil, F.N. Dahli, A.Z. Md Khudzari, H. Adeli, Adv. Mater. Sci. Eng. 2015, 1-19 (2015)

7. E. Song, J.W. Choi, Nanomaterials 3, 498-523 (2013)

8. J.L. Mu, W.J. Fan, S.Y. Shan, T.W. Hu, Y.M. Wang, Q.M. Jia, Adv. Mater. Res. 650, 249-252 (2013)

9. X. Liang, M. Sun, L. Li, R. Qiao, K. Chen, Q. Xiao, et al. Dalton Trans. 41, 2804-2811 (2012)

10. C. Dhivya, S.A.A. Vandarkuzhali, N. Radha, Arab. J. Chem. (2015)

11. D.J. Hines, D.L Kaplan, Crit. Rev. Ther. Drug Carrier Syst. 30, 257-276 (2013)

12. T. Ponnusamy, L.B. Lawson, L.C. Freytag, D.A. Blake, R.S. Ayyala, V.T. John, Biomatter 2, 7786 (2012)

13. S.S. Shin, M. Yoon, J. Ethnopharmacol. 210, 80-87 (2018)

14. T. Ramesh, S.W. Kim, S.Y. Hwang, S.H. Sohn, S.K. Yoo, S.K. Kim, Nutr. Res. 32, 718-726 (2012)

15. S.Y. Lee, J.J. Jeong, S.H. Eun, D.H. Kim, Eur. J. Pharmacol. 762, 333-343 (2015)

16. S.K. Lukman, S. Saidin, Z.K. Lo, R.H. Al-Ashwal, Paper presented at the Proceedings of the 2017 International Conference on Computational Biology and Bioinformatics 101-104 (2017)

17. F. Lewis, M. Cloutier, P. Chevallier, S. Turgeon, J.J. Pireaux, M. Tatoulian, et. Al, ACS Appl. Mater. Interfaces, 3, 2323-2331 (2011)

18. Y. Wang, X. Guo, R. Pan, D. Han, T. Chen, Z. Geng, et al, Mater. Sci. Eng. C 53, 222-228 (2015)

19. N.P. Chauhan, R. Ameta, R. Ameta, S.C. Ameta, Indian J. Chem. Technol. 18, 118-122 (2011)

20. M. Haïdopoulos, S. Turgeon, C. Sarra-Bournet, G. Laroche, D. Mantovani, J. Mater. Sci. Mater. Med. 17, 647-657 (2006)

21. A. Sarvi, A.B. Silva, R.E. Bretas, U. Sundararaj, Polym. Int. 64, 1262-1267 (2015) 